\title{
Telehealth for High-Risk Pregnancies in the Setting of the COVID-19 Pandemic
}

Aleha Aziz, MD, MPH ${ }^{1} \quad$ Noelia Zork, MD ${ }^{1} \quad$ Janice J. Aubey, MD, MPH ${ }^{1} \quad$ Caitlin D. Baptiste, MD Mary E. D’Alton, MD ${ }^{1} \quad$ Ukachi N. Emeruwa, MD, MPH ${ }^{1} \quad$ Karin M. Fuchs, MD, MHA ${ }^{1} \quad$ Dena Goffman, MD ${ }^{1}$ Cynthia Gyamfi-Bannerman, MD, MS ${ }^{1}$ Jennifer H. Haythe, MD ${ }^{2}$ Anita P. LaSala, MD ${ }^{1}$ Nigel Madden, MD, MS ${ }^{1}$ Eliza C. Miller, MD ${ }^{3}$ Russell S. Miller, MD ${ }^{1}$ Catherine Monk, PhD $D^{1,4,5}$ Leslie Moroz, MD ${ }^{1}$ Samsiya Ona, MD ${ }^{1}$ Laurence E. Ring, MD ${ }^{6}$ Jean-Ju Sheen, MD ${ }^{1}$ Erica S. Spiegel, MS ${ }^{1}$ Lynn L. Simpson, MD ${ }^{1} \quad$ Hope S. Yates, JD, MPH ${ }^{1} \quad$ Alexander M. Friedman, MD, MPH ${ }^{1}$

${ }^{1}$ Department of Obstetrics and Gynecology, Vagelos College of Physicians and Surgeons, Columbia University, New York, New York

${ }^{2}$ Division of Cardiology, Department of Medicine, Vagelos College of Physicians and Surgeons, Columbia University, New York, New York

${ }^{3}$ Department of Neurology, Vagelos College of Physicians and Surgeons, Columbia University, New York, New York

${ }^{4}$ Department of Psychiatry, Vagelos College of Physicians and

Surgeons, Columbia University, New York, New York

${ }^{5}$ New York State Psychiatric Institute, New York, New York

${ }^{6}$ Department of Anesthesiology, Vagelos College of Physicians and

Surgeons, Columbia University, New York

Am J Perinatol 2020;37:800-808.
Address for correspondence Alexander M. Friedman, MD, MPH, Division of Maternal-Fetal Fetal Medicine, Department of Obstetrics and Gynecology, Vagelos College of Physicians and Surgeons, Columbia University, 622 West 168th Street, New York, NY 10032 (e-mail: amf2104@cumc.columbia.edu).

\begin{abstract}
Keywords

- coronavirus

- COVID-19

- telehealth

- prenatal care

As New York City became an international epicenter of the novel coronavirus disease 2019 (COVID-19) pandemic, telehealth was rapidly integrated into prenatal care at Columbia University Irving Medical Center, an academic hospital system in Manhattan. Goals of implementation were to consolidate in-person prenatal screening, surveillance, and examinations into fewer in-person visits while maintaining patient access to ongoing antenatal care and subspecialty consultations via telehealth virtual visits. The rationale for this change was to minimize patient travel and thus risk for COVID-19 exposure. Because a large portion of obstetric patients had underlying medical or fetal conditions placing them at increased risk for adverse outcomes, prenatal care telehealth regimens were tailored for increased surveillance and/or counseling. Based on the incorporation of telehealth into prenatal care for high-risk patients, specific recommendations are made for the following conditions, clinical scenarios, and services: (1) hypertensive disorders of pregnancy including preeclampsia, gestational hypertension, and chronic hypertension; (2) pregestational and gestational diabetes mellitus; (3) maternal cardiovascular disease; (4) maternal neurologic conditions; (5) history of preterm birth and poor obstetrical history including prior stillbirth; (6) fetal conditions such as intrauterine growth restriction, congenital anomalies, and multiple gestations including monochorionic placentation; (7) genetic counseling; (8) mental health services; (9) obstetric anesthesia consultations; and (10) postpartum care. While telehealth virtual visits do not fully replace in-person encounters during prenatal
\end{abstract}

received

April 23, 2020

accepted after revision

April 24, 2020

published online

May 12, 2020
Copyright $\odot 2020$ by Thieme Medical

Publishers, Inc., 333 Seventh Avenue, New York, NY 10001, USA Tel: $+1(212) 760-0888$.
DOI https://doi.org/ 10.1055/s-0040-1712121. ISSN 0735-1631. 
care, they do offer a means of reducing potential patient and provider exposure to COVID-19 while providing consolidated in-person testing and services.

Key Points

- Telehealth for prenatal care is feasible.

- Telehealth may reduce coronavirus exposure during prenatal care.

- Telehealth should be tailored for high risk prenatal patients.

The World Health Organization declared the novel coronavirus disease 2019 (COVID-19) outbreak as a public health emergency of international concern on January 30, 2020, and on March 11, 2020, it was characterized as a pandemic. In New York City, the number of infections increased exponentially, leading to the city becoming an international epicenter of COVID-19 infection with a declaration of a state disaster emergency by the New York governor on March 7, 2020. By the third week in March, an executive order in New York State was announced encouraging social distancing and limiting gatherings. ${ }^{1}$ For obstetric patients receiving prenatal care in the greater New York City metropolitan area, attending inperson prenatal care visits with potential interaction with other patients, health care personnel, and staff represented an additional source of potential exposure.

Telehealth represents one approach to optimizing care and mitigating risk for exposure for prenatal patients in the setting of the COVID-19 pandemic. Telehealth previously had been proposed as a means of overcoming obstacles associated with providing medical care in the setting of disasters. ${ }^{2}$ Prior research on integration of telemedicine into the health care system has been conducted in rural communities and has demonstrated both opportunities for improved care and challenges. ${ }^{3,4}$ Telehealth may be used across medical specialties as a means of maintaining patient access to care in the setting of COVID-19 while facilitating physical distancing. ${ }^{5}$

Given that many other locales in the United States may be faced with similar challenges related to the COVID-19 pandemic, the purpose of this manuscript is to review the adoption of telehealth for high-risk obstetric patients receiving care at two hospitals and an affiliated clinic system in New York City.

\section{Materials and Methods}

Columbia University Irving Medical Center-affiliated obstetric ambulatory prenatal care facilities located in midtown Manhattan, Washington Heights, and Westchester County transitioned to telehealth appointments for prenatal care during the COVID-19 outbreak in the greater New York City metropolitan area. These facilities provide care for patients with both public and commercial insurance. Patients accessing prenatal care at these sites deliver at New York-Presbyterian Morgan Stanley Children's Hospital, a tertiary referral center performing approximately 4,600 deliveries per year, and New York Presby- terian Allen Hospital, an associated community hospital performing approximately 2,300 deliveries per year.

Telehealth was incorporated into the provision of prenatal care at an accelerated rate beginning March 17, 2020, shortly after schools, bars, and restaurants in New York City were ordered to close. Prenatal care regimens were developed individualizing care to patient risk factors and incorporating telehealth formats from prior studies and expert recommendations, including resources from the American College of Obstetricians and Gynecologists and the Society for Maternal-Fetal Medicine. ${ }^{6-10}$ Virtual visits were performed with Epic Haiku and Canto, with patients accessing appointments via the Epic Connect and MyChart application on their mobile phones or tablets. Providers were trained in this software 1 week prior to adoption via a short video tutorial course ( $\sim 15$ minutes in duration) and a user guide with additional support as needed. Patients were guided through installation of software by staff in person, by emailed instructions, and/or remotely by telephone guidance.

To understand how prenatal care was formatted for telehealth in practice and then specifically tailored for conditions placing patients at higher risk for adverse maternal or neonatal outcomes, we queried obstetrician gynecologists, maternal-fetal medicine specialists (including fellows and attending physicians), medical consultants including cardiologists, neurologists, obstetric anesthesiologists, women's health psychologists, and genetic counselors at Columbia University who were involved in this rapid transition to telehealth. We reviewed surveillance and management algorithms to determine how telehealth use was applied to prenatal care. The final management recommendations were created by an iterative process and edited to achieve consensus. In addition to recommendations for general prenatal care, specific recommendations were made for the following conditions, clinical scenarios, and subspecialty services: (1) hypertensive disorders of pregnancy including preeclampsia, gestational hypertension, and chronic hypertension; (2) pregestational and gestational diabetes mellitus; (3) maternal cardiovascular disease; (4) maternal neurologic conditions; (5) history of preterm birth and poor obstetrical history, including prior stillbirth; (6) fetal conditions such as intrauterine growth restriction (IUGR), congenital anomalies, and multiple gestations, including monochorionic placentation; (7) genetic counseling; (8) mental health services; (9) obstetric anesthesia consultations; and (10) postpartum care. 


\section{Results}

The general approach for applying telehealth to prenatal care involved consolidating testing and services that needed to be performed in-person while performing counseling during telehealth sessions. The proposed framework for telehealth prenatal care eliminates approximately one-half of in person visits for low-risk patients (-Table 1 ). If the patient is

\begin{tabular}{|c|c|c|c|}
\hline GA & Visit type & Counseling and education & Associated evaluation \\
\hline Intake & Virtual & $\begin{array}{l}\text { - Clinical history taken } \\
\text { - Counseling on nutrition, weight gain, risk factors } \\
\text { based on environment and comorbidities } \\
\text { - Genetic screening/testing options } \\
\text { - Orientation to practice } \\
\text { - Prenatal vitamins prescription }\end{array}$ & \\
\hline $11-13 w k$ & In-person & $\begin{array}{l}\text { - Blood pressure cuff teaching } \\
\text { - If applicable, genetic counselor appointment and } \\
\text { screening/testing }\end{array}$ & $\begin{array}{l}\text { - Physical exam } \\
\text { - Prenatal laboratories } \\
\text { - Dating ultrasound } \\
\text { ( } \pm \text { nuchal translucency) }\end{array}$ \\
\hline $14-17$ wk & Virtual & $\begin{array}{l}\text { - Review of systems including mental health symp- } \\
\text { toms and intimate partner violence screening } \\
\text { - Prenatal laboratory and genetic screening review }\end{array}$ & - Home blood pressure and weight \\
\hline $18-22$ wk & In-person & - Review of systems & $\begin{array}{l}\text { - Vital signs including weight } \\
\text { - Urine evaluation } \\
\text { - Anatomy ultrasound }\end{array}$ \\
\hline $23-26 w k$ & Virtual & $\begin{array}{l}\text { - Review of systems } \\
\text { - Counseling on gestational diabetes screening and } \\
\text { Tdap vaccine }\end{array}$ & - Home blood pressure and weight \\
\hline $27-28 w k$ & In-person & $\begin{array}{l}\text { - Review of systems } \\
\text { - Kick-count counseling }\end{array}$ & $\begin{array}{l}\text { - Vital signs including weight } \\
\text { - Doppler heart tones } \\
\text { - Urine evaluation } \\
\text { - Diabetes screening } \\
\text { - 3rd trimester laboratory work } \\
\text { - Tdap vaccine } \\
\text { - Fundal height evaluation } \pm \text { growth ultra- } \\
\text { sound } \\
\text { - RhoGAM, if indicated }\end{array}$ \\
\hline $29-35$ wk & $\begin{array}{l}2 \text { or } 3 \\
\text { virtual } \\
\text { visits }\end{array}$ & $\begin{array}{l}\text { - Review of systems } \\
\text { - Delivery counseling (TOLAC, if applicable) } \\
\text { - Breastfeeding education } \\
\text { - Preterm labor and preeclampsia precautions } \\
\text { - Counseling on COVID-19 labor and delivery specific } \\
\text { protocol, anticipatory guidance, postpartum care } \\
\text { - Car seat, pediatrician education } \\
\text { - Family planning counseling }\end{array}$ & - Home blood pressure and weight \\
\hline 36 wk & In-person & $\begin{array}{l}\text { - Labor precautions } \\
\text { - ECV planning if applicable. } \\
\text { - Planning for timing of delivery, induction (both } \\
\text { indicated and elective), and late-term and post- } \\
\text { dates testing, if indicated }\end{array}$ & $\begin{array}{l}\text { - Vital signs including weight } \\
\text { - Urine evaluation } \\
\text { - Fundal height } \pm \text { growth ultrasound } \\
\text { - Doppler heart tones } \\
\text { - GBS screening }\end{array}$ \\
\hline \multicolumn{4}{|c|}{$\begin{array}{l}\text { Care from } 36 \text { wk on can modified to weekly in-person visits, based on risk; otherwise for weeks without in-person visit, } \\
\text { virtual visit should be scheduled }\end{array}$} \\
\hline $37-38 w k$ & 2 virtual & - Labor precautions & - Home blood pressure and weight \\
\hline $39 w k$ & In-person & - Labor precautions & $\begin{array}{l}\text { - Vital signs including weight } \\
\text { - Urine evaluation } \\
\text { - Fundal height } \pm \text { growth ultrasound } \\
\text { - Doppler heart tones }\end{array}$ \\
\hline $40 \mathrm{wk}$ & In-person & - Labor precautions & $\begin{array}{l}\text { - Vital signs including weight } \\
\text { - Urine evaluation } \\
\text { - Doppler heart tones } \\
\text { - Fetal testing if indicated } \\
\text { - Fundal height evaluation }\end{array}$ \\
\hline
\end{tabular}

Abbreviations: COVID-19, novel coronavirus disease 2019; ECV, external cephalic version; GA, gestational age; GBS, Group B streptococcus; TOLAC, trial of labor after cesarean. 
presumed to be $<11$ weeks of gestation at the start of care, the first visit is virtual with the clinical history obtained, the patient oriented to the clinical practice, and genetic screening options reviewed. The subsequent visit at 11 to 14 weeks of gestation is the initial in-person encounter, and at this visit a physical examination and prenatal blood work are performed, along with genetic screening and a first trimester ultrasound to confirm dating if not performed earlier. Chorionic villus sampling can be performed at this visit, if desired. The next visit is performed virtually approximately 4 weeks later, followed by an in-person visit at 18 to 22 weeks to coincide with the fetal anatomy ultrasound. The subsequent visit is virtual, followed by an in-person visit at 27 to 28 weeks to perform third trimester blood work including screening for gestational diabetes mellitus, to assess fundal height, and to administer the Tdap vaccine and RhoGAM, if indicated. The subsequent two visits are virtual, and at 36 weeks an in-person visit is again performed to assess fundal height and screen for group $B$ streptococcus colonization. Blood pressures should be regularly measured and logged by patients at home if they are not attending in-person visits. Based on the patient's risk profile, either telehealth or in-person visits may occur at 37 and 38 weeks, with resumption of in-person visit at 39 weeks.

For telehealth visits, providers should wear professional attire and sit in front of a simple, nondistracting background with access to the patient's medical record. Patients should schedule their visit during a time when they can be alone in a quiet room and are encouraged to avoid multitasking (e.g., actively working, driving a car, or walking), as this creates distractions for both parties and can cause safety and connectivity issues. An interpreter, if needed, can be accessed via speaker phone. Patient privacy is a concern during telehealth visits and patients should be encouraged to find a private setting where they can either be alone or with a support person of their choosing. Certain populations may not have a device with video capabilities (smart phone or tablet) for economic or cultural reasons, in which case a telephone visit has been an alternative. Telehealth visit scheduling should take into account the possibility that virtual visits may take longer than an in-person visit. When a woman's prenatal care is transitioned from in-person to remote care visits, she may feel that she is not receiving the same access to medical care. It is important to convey to the patient that although much of her care will be virtual, if any concerns are not addressed remotely she can present for an in-person assessment and that her obstetrical care team will remain available to answer questions in person.

Certain high-risk pregnancies may require increased frequency of in-person visits, whether with the obstetric care provider, nonobstetric consultants, or both. In many cases, fetal testing required in high-risk pregnancies to decrease risk for adverse outcomes will need to be preserved, perhaps with institution-specific modifications due to the COVID-19 pandemic. Below are considerations for integration of telemedicine into the care of pregnant women with high-risk maternal or fetal conditions.

\section{Specific Considerations for Telehealth Management for High-Risk Pregnant Patients}

\section{Hypertensive Disorders of Pregnancy}

For patients with chronic or gestational hypertension, preeclampsia, or risk factors for the development of hypertensive disease in pregnancy, the key resource in facilitating the provision of prenatal care virtually is a home automated blood pressure cuff that the patient is comfortable using (-Table 2). While a home blood pressure cuff should be available to all women receiving telehealth care, frequent and proper cuff use is particularly important for women with hypertensive disorders of pregnancy. Patients should be able to report blood pressure data to providers in a timely manner. Patients should be trained as early as possible during an in-person visit to measure their blood pressure, and their cuff results should be compared with the office measurement to ensure calibration. Additionally, to confirm proper use patients can perform the blood pressure measurement during the video visit with the provider observing. Many of these patients will begin fetal growth monitoring and testing in the third trimester. To decrease office encounters, medical assistants may obtain patient vital signs in the ultrasound units so that providers have an additional source of information regarding blood pressure status. For blood pressures elevated beyond specific thresholds, medical assistants can alert the provider to determine further management. As hypertension in pregnancy may worsen in the late third trimester, patients may be seen weekly in person from 36 weeks onward. The exact format of inperson prenatal care may be determined by the specific clinical details and social circumstances of the patient.

\section{Pregestational and Gestational Diabetes}

Education is the mainstay of diabetes management pregnancy, which lends itself well to telehealth visits ( - Table 2). For women with gestational diabetes mellitus, the appropriate testing supplies are sent to their pharmacy and diabetes selfmanagement education is provided by a certified diabetes educator during a virtual visit. Diabetes educational materials are e-mailed to the patient as additional resources for them to review independently. ${ }^{11}$ Patients are additionally provided virtual visits with a registered dietician. Patients may use a paper log or a phone application (e.g., mySugr) to document their blood glucose values. The patient is scheduled for a telehealth visit with a maternal-fetal medicine specialist to review her glucose log and review the plan of care for the remainder of her pregnancy 1 to 2 weeks after receiving diabetes self-management education and nutrition counseling. If the patient is prescribed insulin and requires education on insulin administration, a telehealth visit is performed with the certified diabetes educator or a link to an instructional video is e-mailed to the patient. ${ }^{11}$ Virtual visits offer an advantage over an instructional video, as they allow the certified diabetes educator to verify that the patient is administering the insulin correctly. E-mail or phone communication is used on a weekly basis to review blood glucose measurements. These same workflows can also be used for patients with pregestational diabetes mellitus. Similar to patients with 
Table 2 Key telehealth management points for high-risk pregnancies

\begin{tabular}{|c|c|}
\hline High-risk condition & Key management points \\
\hline $\begin{array}{l}\text { Hypertensive } \\
\text { disorders of pregnancy }\end{array}$ & $\begin{array}{l}\text { - A home blood pressure cuff is a key resource for management } \\
\text { - Vital signs can be measured at fetal testing and growth ultrasounds } \\
\text { - Weekly in-person visits may be preferable from } 36 \text { wk gestation }\end{array}$ \\
\hline $\begin{array}{l}\text { Diabetes } \\
\text { (pregestational } \\
\text { and gestational) }\end{array}$ & $\begin{array}{l}\text { - Diabetes education may occur with dietician, diabetes educator, and provider via video visits } \\
\text { - Video and online diabetes information can be sent to the patient for review } \\
\text { - Remote monitoring of blood glucose with weekly email or phone review is recommended } \\
\text { - Vital signs can be measured at fetal testing and growth ultrasounds }\end{array}$ \\
\hline Cardiovascular disease & $\begin{array}{l}\text { - A home blood pressure cuff is a key resource for management as is having a home scale to } \\
\text { measure weight } \\
\text { - In-person visits may be preferred from } 34 \text { wk gestation } \\
\text { - Co-management with a cardiologist with monthly virtual visits for higher-risk diagnoses may } \\
\text { be indicated } \\
\text { - One or more maternal echocardiograms may be indicated during the pregnancy }\end{array}$ \\
\hline $\begin{array}{l}\text { Neurologic } \\
\text { conditions }\end{array}$ & $\begin{array}{l}\text { - Co-management with neurologist with virtual visits in the first and third trimester may be } \\
\text { indicated } \\
\text { - A family member may be helpful in assisting with a virtual neurology exam } \\
\text { - Concerning neurologic symptoms require prompt in-person evaluation }\end{array}$ \\
\hline $\begin{array}{l}\text { History of preterm } \\
\text { birth and poor } \\
\text { obstetrical history }\end{array}$ & $\begin{array}{l}\text { - Absent a cerclage, cervical length screening should continue as indicated } \\
\text { - If a cerclage is present cervical length screening may be discontinued } \\
\text { - There should be a low threshold for in-person evaluation of concerning symptoms } \\
\text { - Modifications of fetal testing for prior stillbirth are not recommended }\end{array}$ \\
\hline $\begin{array}{l}\text { Fetal conditions } \\
\text { (IUGR, congenital anomalies, } \\
\text { and multiple gestations) }\end{array}$ & $\begin{array}{l}\text { - A home blood pressure cuff is a key resource for management } \\
\text { - No modification of fetal testing is recommended for conditions with increased risk for } \\
\text { stillbirth } \\
\text { - Patient privacy related to termination counseling may be a particular concern during } \\
\text { - Inlehealth visits }\end{array}$ \\
\hline Genetic counseling & $\begin{array}{l}\text { - Counseling may be performed virtually with visual aids and online resources } \\
\text { - Genetic counselors may manage invasive testing and blood samples remotely } \\
\text { - Carrier screening saliva kits may be sent to patients' homes }\end{array}$ \\
\hline Mental health services & $\begin{array}{l}\text { - Virtual visits may be conducted with acknowledgment that the experience is different than } \\
\text { in-person encounters }\end{array}$ \\
\hline Obstetric anesthesia & $\begin{array}{l}\text { - Virtual visits may be performed with airway exam } \\
\text { - Telehealth discussion focuses on eligibility for labor analgesia and timing } \\
\text { - In-person evaluation occurs on inpatient admission with exam of airway and spine }\end{array}$ \\
\hline Postpartum care & $\begin{array}{l}\text { - Routine postpartum visits including post-cesarean may be performed virtually unless there } \\
\text { are acute symptoms } \\
\text { - Lactation consultations may be performed virtually } \\
\text { - A home blood pressure cuff is a key resource for management }\end{array}$ \\
\hline
\end{tabular}

Abbreviation: IUGR, intrauterine growth restriction.

hypertensive disorders of pregnancy, blood pressures may be measured at the ultrasound unit or nonstress test visit and conveyed to providers, as appropriate.

\section{Cardiovascular Disease}

Determination as to whether a patient with an underlying cardiac condition can be transitioned to virtual visits depends on the specific underlying diagnosis, input from the cardiology consultant, and the patient's clinical history including hospitalizations and symptomatology. If the patient remains asymptomatic and has not required recent hospitalizations, it is unlikely that she will require many additional in-person prenatal visits with the obstetrician beyond those scheduled in routine, telehealth-formatted prenatal care before 34 weeks ( - Table 1 ) unless new symptoms or findings develop. Since patients with cardiovascular disease are more likely to have worsening symptoms with advancing gestational age, they should be seen in-person from 34 weeks onward by the obstetrician. An assessment of the patient's cardiovascular status during her physical examination at the initial in-person prenatal visit may inform the provider regarding whether the patient's heart disease is well-compensated and what her risk for possible complications is during the course of pregnancy. Thereafter, patients can report any change in symptoms or baseline physical activity during a virtual visit and be triaged accordingly. In addition to a blood pressure cuff, having a scale at home is particularly important for prenatal patients with cardiovascular disease.

Most patients with cardiovascular comorbidities are comanaged during pregnancy with a cardiologist. Many cardiologists in our system have similarly converted in-person 
appointments into telehealth visits. Patients with congenital heart disease, valvular disease, cardiomyopathy, or a history of acute coronary syndrome should have telehealth visits at least monthly with their cardiologist (-Table 2 ). If patients are symptomatic from any of the aforementioned conditions, they should be seen in-person by their cardiologist. For cardiomyopathy, a baseline echocardiogram is essential with possible repeat evaluation based on clinical characteristics, symptoms, and recommendation of the cardiologist. One or more maternal echocardiogram studies may be required for other cardiac conditions. If antihypertensive medications need to be titrated by the cardiologist due to elevated blood pressures, virtual encounters should be conducted weekly until blood pressures are under control, at which point visits with the cardiologist can be spaced out unless blood pressure changes occur. However, if a cardiologist suspects a patient is developing gestational hypertension or preeclampsia, recommendations should be deferred to the obstetric provider and the patient will likely require an in-person evaluation by the obstetrical team for further management. For patients who require laboratory visits (to determine anticoagulation status, for example), these appointments can be coordinated with other care such as maternal echocardiogram or fetal ultrasound studies. If these patients are undergoing any third-trimester ultrasounds or nonstress testing, appointments should be coordinated with in-person prenatal care visits. Providers caring for pregnant women with cardiovascular disease should be flexible and reactive to the patient's symptoms and overall condition.

\section{Neurologic Conditions}

A standard neurology consultation includes a careful history, a detailed physical examination, and a review of neuroimaging. Vascular neurologists already maybe experienced with telemedicine evaluations if they perform "tele-stroke" evaluations in emergency rooms that do not have in-house neurologists. While neurologic consultation can often be reformatted for virtual visits, patients with focal or acute onset symptoms or symptoms that are particularly concerning (e.g., "the worst headache of my life") should be evaluated expediently in-person in an emergency room or triage setting. For some patients complaining of a headache, the fundoscopic examination is of critical importance. If "red flag" elements are elicited from the history and examination (positionality of headache, blurred vision, and visual field deficits), the patient should be seen in-person for fundoscopy either in an ophthalmology office, urgent care, or emergency room setting.

During pregnancy, most patients with preexisting cerebrovascular disease can be seen by the neurologist virtually once during the first trimester and then again for delivery planning in the third trimester. A family member assisting the patient may facilitate virtual visits, for example, evaluation of gait may require a camera to be held at a distance. In addition, some elements of the sensory exam will require assistance from a helper, who can be instructed by the neurologist. Cardiac and carotid auscultation cannot be performed during a telemedicine visit and should be performed at the next in-person visit.

\section{History of Preterm Birth and Poor Obstetrical History Including Prior Stillbirth}

Patients with a history of preterm birth and poor obstetrical history may experience significant anxiety related to risk of recurrence during a subsequent pregnancy. Telehealth is well suited for counseling such patients throughout pregnancy. If patients require a history-indicated cerclage, coordination of care for this procedure can be done remotely. Cervical length screening for patients with a history of prior preterm birth should be continued as indicated. If a patient undergoes cerclage placement, continuation of cervical length screening should be discontinued due to its minimal potential to alter subsequent management. Patients can be brought into the office for a speculum or vaginal examination as dictated by patient symptoms. Otherwise, prenatal care can proceed via the standard telehealth framework with a low threshold for reporting and evaluation of concerning symptoms. If patients are receiving intramuscular progesterone, nursing services, or self-administration education should be arranged for home receipt. In the setting of prior stillbirth, the benefits of fetal testing may outweigh risks related to COVID-19 exposure and patient care should be individualized.

\section{Fetal Conditions}

Prenatal appointments for healthy women with fetal conditions can often be managed using the standard telehealth framework (-Table 1) without an increase in the frequency for in-person visits. For those at increased risk for preeclampsia or mirror syndrome, blood pressures can be measured by patients during routine video visits, and also between encounters. In these cases, blood pressure logs maintained by patients should be sent electronically and reviewed weekly by medical staff with precautions that patients notify the office by telephone should the threshold blood pressure levels be reached (e.g., 140/90 mm Hg). In the setting of a fetal anomaly not associated with amniotic fluid abnormalities (oligohydramnios and polyhydramnios), IUGR, or other features that increase the risk of stillbirth, fetal testing and ultrasound evaluation can be individualized. A concern in conducting telehealth visits in the setting of a major fetal anomaly is patient privacy, particularly if the patient is considering pregnancy termination. Even if patients are encouraged to find a private setting for virtual visits, telemedicine can present challenges in preserving confidentiality as providers cannot control who else is present in the patient's environment. It is necessary to plan ahead for consultation about complex fetal conditions via telehealth to ensure that patients can have the presence of the support people of their choosing during critical and/or time-sensitive discussions.

For fetal conditions where there is clear increased risk of stillbirth, such as IUGR, antenatal testing should continue as indicated. The performance of routine umbilical artery Doppler ultrasound for pregnancies complicated by IUGR may provide important information that guides clinical management. For dichorionic twin pregnancies with normal fetal growth, fetal testing can be individualized based on other 
obstetric and clinical factors. However, for monochorionic twin pregnancies, close ultrasound surveillance on an alternate week basis from 16 weeks onward should continue due to the high prevalence for placenta-related complications including twin-twin transfusion syndrome, unequal placental sharing, and fetal demise. In centers like ours where ultrasound units are in the same facility or in close proximity to prenatal care offices, patients can coordinate in-person provider visits on the same day as their ultrasound appointment. Over the course of the pandemic, the number of patients in waiting rooms has decreased significantly due to increased telehealth visits, decreased visits for gynecological indications, and increased spacing of in-person visits for prenatal care and ultrasound, thereby facilitating physical distancing.

\section{Genetic Counseling}

During the rapid transition to telemedicine, genetic counselors have modified consults to increase effectiveness of counseling in a virtual space. In our practice, genetic counselors are no longer on site, conducting patient consultations remotely via telehealth. Use of visual aids for patient education are presented by either placing the aid in front of the camera for the patient or sharing electronic images. As genetic counselors are no longer working on site, the handling of chorionic villi, amniotic fluid, and blood samples must also be coordinated remotely ensuring safe delivery of the samples to the laboratory for genetic testing. This has required the creation of new prenatal sample management protocols, increased support from the on-site team (medical assistants, research assistants, and nursing), and clear communication between the genetic counselors and support staff. Blood draws are scheduled for testing which cannot utilize at home saliva kits, such as noninvasive prenatal testing (NIPT), and the best attempts are made to coordinate the blood draw with a preexisting ultrasound appointment to minimize additional visits to the hospital. Saliva kits for expanded carrier screening are sent directly to patients' homes from the performing laboratories.

\section{Mental Health}

It is customary for obstetric patients in our system requiring mental health services to have an in-person encounter with the psychologist, social worker, or psychiatric nurse practitioner before transitioning to telehealth visits. This establishes an alliance between provider and patient, and ensures that the mental health provider is accurately evaluating her needs and acuity of her issues. Initial in-person visits also help set the tone for the relationship by establishing privacy and prioritizing the patient's concerns. If there is concern for domestic violence or an abusive relationship in the home environment, it is important to directly address these issues during the inperson session and plan accordingly. With the advent of almost exclusive telehealth mental health services during the pandemic, acknowledging the limitations and specific challenges of virtual visits, as well as addressing the above concerns without the in-person context, has been important for both patients and providers. Finally, as is standard for mental health care, it is essential to ensure that the patient has the information needed to contact her provider, or someone on the mental health team, in the case of urgent concerns, and it is critical that the patient understands and agrees to the criteria for calling 911 for a mental health emergency.

\section{Obstetric Anesthesia}

Some patients may have medical, obstetrical, or surgical factors that require additional planning by obstetric anesthesiologists for analgesia during the delivery hospitalization. This planning may occur prior to the delivery admission during an obstetric anesthesia consultation. Obstetric anesthesia consultations generally focus on counseling and involve only a limited physical examination. Given that the review of clinical history and imaging are often more important than examination findings in determining management, the biggest challenge of obstetric anesthesia telehealth consultations can be obtaining medical records which may be in the patient's possession and not available on a digital medical record platform. The physical examination elements of the in-person obstetric anesthesia consultation include airway assessment and examination of the lumbar spine. The former may be performed virtually, but the latter requires an inperson evaluation. Fortunately, the findings on these exams seldom change the anesthesia management, as general anesthesia is rarely planned. Once a patient presents for delivery, an airway assessment is still performed, regardless of whether the evaluation previously occurred virtually. Additionally, even if scoliosis or other clinical factors make neuraxial anesthesia more difficult, regional anesthesia is almost always attempted. As some of the most critical parts of the obstetric anesthesia consultation involve determining if the patient can have neuraxial labor analgesia and whether she should have anesthesia administered early in labor, this planning translates well into a discussion between the anesthesiologist and patient via telehealth. Additional features of this conversation can include discussion regarding additional invasive monitoring, anesthesia for cesarean, and contingency planning if neuraxial anesthesia cannot be achieved.

\section{Postpartum Care}

A full physical examination in the office during the postpartum period is preferable absent pandemic conditions. However, in the setting of COVID-19, routine postpartum appointments for both vaginal and cesarean deliveries can be done virtually if the patient is stable without abnormal pain or bleeding. Healing of a cesarean skin incision can be evaluated by the patient showing the provider the incision with the camera from a smartphone or other device. For patients with hypertensive diseases of pregnancy, virtual postpartum visits 1 week after delivery can be performed to review blood pressures and medical management. If bloodwork is necessary, new symptoms are present, or blood pressure is poorly controlled, an in-person visit may be required. Postpartum breastfeeding consultations can also be performed virtually. Patients with high-risk cardiovascular and noncardiovascular medical conditions, as well as other major comorbidities may require in-person visits with prompt referral to an emergency room or triage for 
concerning findings or symptoms. Telehealth can be used as a means of achieving the American College of Obstetricians and Gynecologists' goals for ongoing postnatal care rather than a single encounter in the postpartum period. ${ }^{12}$ An early telehealth visit does not preclude a possible later (within 12 weeks) postpartum visit in the setting of improved pandemic conditions.

\section{Discussion}

The American College of Obstetricians and Gynecologists notes that telehealth is increasingly used in obstetrics and that obstetrician-gynecologists should consider becoming familiar with this new technology. ${ }^{13}$ The potential benefits of virtual visits in the setting of the COVID-19 pandemic may further support provision of telehealth services despite well characterized legal, regulatory, liability, and reimbursement hurdles. ${ }^{13}$ Prior research has demonstrated comparable outcomes with prenatal telehealth visits, ${ }^{14}$ with patients demonstrating satisfaction with virtual visits. ${ }^{15}$ A recent systematic review of telehealth interventions in obstetrics and gynecology evaluated 47 studies of which 19 pertained to low-risk obstetrics and 13 pertained to high-risk obstetrics. Findings included that patient-generated data transmitted remotely led to fewer scheduled outpatient visits for the management of diabetes and hypertension, ${ }^{16}$ providing supportive evidence for telehealth use in selected high-risk obstetric scenarios. Overall, the authors found limited rigorous evidence supporting telehealth interventions in obstetrics and gynecology, although some interventions did appear promising for clinical uptake and future study. ${ }^{16}$

This set of recommendations does not provide any definitive evidence regarding the relative benefits of telehealth beyond the inference that prenatal care can be organized to consolidate in-person visits and maintain surveillance and routine interventions while performing counseling during virtual appointments, thus reducing the risk for COVID-19 exposure. In addition to reducing the risk of exposure, there may be additional social and economic benefits of telehealth-consolidated prenatal care for women who, in the setting of the COVID-19 pandemic, are working remotely, have increased child-care responsibilities, or both. There is no data available regarding whether the format recommended here is better or worse from a comparative effectiveness standpoint than other prenatal telehealth regimens. Given that severe respiratory infections have been documented among pregnant women with COVID-19, it is reasonable to presume that there is some benefit in reducing risk of exposure particularly in locales with a high prevalence of the virus such as New York City. ${ }^{17,18}$ The prevalence of COVID-19 in an individual locale may determine whether the theoretical benefits of transitioning to telehealth for prenatal care outweigh the logistical challenges of implementation and potential small risks of using abbreviated in-person prenatal care regimens that have not been validated in large populations. It is likely that the recommendations presented here will be further refined and updated as more data on COVID-19 and the comparative effectiveness of telehealth prenatal care becomes available. Further qualitative and survey data on provider experience with telehealth along with quantitative data on the operational transition to telehealth at our center is planned for a separate analysis. Outpatient management of prenatal care patients with confirmed COVID-19 infections not requiring hospitalizations is also presented in a separate analysis. Strengths of these recommendations include the real-world clinical implementation in the setting of a COVID-19 pandemic epicenter, the relative speed of implementation, the feasibility in a variety of settings within our health system, and the specialization for a large subset of women at high risk for adverse neonatal and maternal outcomes.

Obstetricians should explain the rationale for any change in prenatal care scheduling with their patients, discuss alternate modalities, such as telemedicine, and document the aforementioned patient-physician conversations in the medical record. In addition to telehealth, important principles for preventing COVID-19 spread include spacing out inperson appointments for asymptomatic patients to decrease the number of people in the clinical space simultaneously and limiting the number of times that patients are asked to leave their homes. While protecting pregnant women from exposure to COVID-19 was the primary objective of our conversion to telehealth, other benefits of telehealth include reducing provider exposures and reduced use of personal protective equipment in the context of recognized shortages.

\section{Conclusion}

In conclusion, while telehealth virtual visits do not fully replace in-person encounters during prenatal care, they offer a means of reducing patient and provider exposure to COVID-19 while providing consolidated in-person testing and services. Prenatal care telehealth regimens can be modified for high-risk patients to ensure they receive adequate access to fetal testing and ultrasound examinations, nonobstetric consultants, and their obstetric providers.

Funding

None.

Conflict of Interest

M.E.D. has had a leadership role in the American College of Obstetricians and Gynecologists II's Safe Motherhood Initiative which has received unrestricted funding from Merck for Mothers. The other authors did not report any potential conflicts of interest.

\section{References}

1 New York State on PAUSE. 10 point plan. Available at: https:// coronavirus.health.ny.gov/new-york-state-pause. Accessed April 19, 2020

2 Lurie N, Carr BG. The role of telehealth in the medical response to disasters. JAMA Intern Med 2018;178(06):745-746

3 Gagnon MP, Duplantie J, Fortin JP, Landry R. Implementing telehealth to support medical practice in rural/remote regions: what are the conditions for success? Implement Sci 2006;1:18 
4 Seto E, Smith D, Jacques M, Morita PP. Opportunities and challenges of telehealth in remote communities: case study of the Yukon telehealth system. JMIR Med Inform 2019;7(04):e11353

5 Hollander JE, Carr BG. Virtually perfect? Telemedicine for COVID19. N Engl J Med 2020

6 Butler Tobah YS, LeBlanc A, Branda ME, et al. Randomized comparison of a reduced-visit prenatal care model enhanced with remote monitoring. Am J Obstet Gynecol 2019;221(06):638e1-638e8

7 de Mooij MJM, Hodny RL, O'Neil DA, et al. OB nest: reimagining low-risk prenatal care. Mayo Clin Proc 2018;93(04):458-466

8 The American College of Obstetricians and Gynecologists. Physician FAQs. Available at: https://www.acog.org/clinical-information/physician-faqs/. Accessed April 19, 2020

9 The Society for Maternal-Fetal Medicine. Coronavirus (COVID-19). Available at: https://www.smfm.org/covid19. Accessed April 19, 2020

10 Boelig RC, Saccone G, Bellussi F, Berghella V. MFM guidance for COVID-19. Am J Obstet Gynecol MFM. 2020;19:100106 (e-pub ahead of print). Doi: 10.1016/j.ajogmf.2020.100106

11 Mayo Clinic. How to use an insulin pen: mayo clinic patient education. Available at: https://www.youtube.com/watch?v=_fCqEIn7ccA. Accessed April 19, 2020
12 McKinney J, Keyser L, Clinton S, Pagliano C. ACOG committee opinion no. 736: optimizing postpartum care. Obstet Gynecol 2018;132(03):784-785

13 Implementing Telehealth in Practice. Implementing telehealth in practice. Obstet Gynecol 2020;135(02):e73-e79

14 Pflugeisen BM, McCarren C, Poore S, Carlile M, Schroeder R. Virtual visits: managing prenatal care with modern technology. MCN Am J Matern Child Nurs 2016;41(01):24-30

15 Pflugeisen BM, Mou J. Patient satisfaction with virtual obstetric care. Matern Child Health J 2017;21(07):1544-1551

16 DeNicola N, Grossman D, Marko K, et al. Telehealth interventions to improve obstetric and gynecologic health outcomes: a systematic review. Obstet Gynecol 2020;135(02):371-382

17 Sutton D, Fuchs K, D’Alton M, Goffman D. Universal screening for SARS-CoV-2 in women admitted for delivery. N Engl J Med 2020 (e-pub ahead of print) . Doi: 10.1056/NEJMc2009316

18 Breslin N, Baptiste C, Gyamfi-Bannerman C, et al. Coronavirus disease 2019 infection among asymptomatic and symptomatic pregnant women: Two weeks of confirmed presentations to an affiliated pair of New York City hospitals. Am J Obstet Gynecol MFM 2020:100118 (e-pub head of print). Doi: 10.1016/j.ajogmf.2020.100118. 